\title{
Genetics, sleep and memory: a recall-by- genotype study of ZNF804A variants and sleep neurophysiology
}

Charlotte Hellmich ${ }^{1}$, Claire Durant ${ }^{2}$, Matthew W. Jones ${ }^{1}$, Nicholas J. Timpson ${ }^{3}$, Ullrich Bartsch ${ }^{1^{*+}}$ and Laura J. Corbin ${ }^{3 *+}$

\begin{abstract}
Background: Schizophrenia is a complex, polygenic disorder for which over 100 genetic variants have been identified that correlate with diagnosis. However, the biological mechanisms underpinning the different symptom clusters remain undefined. The rs1344706 single nucleotide polymorphism within ZNF804A was among the first genetic variants found to be associated with schizophrenia. Previously, neuroimaging and cognitive studies have revealed several associations between rs1344706 and brain structure and function. The aim of this study is to use a recall-by-genotype (RBG) design to investigate the biological basis for the association of ZNF804A variants with schizophrenia. A RBG study, implemented in a population cohort, will be used to evaluate the impact of genetic variation at rs1344706 on sleep neurophysiology and procedural memory consolidation in healthy participants.

Methods/Design: Participants will be recruited from the Avon Longitudinal Study of Parents and Children (ALSPAC) on the basis of genotype at rs1344706 $(n=24)$. Each participant will be asked to take part in two nights of in-depth sleep monitoring (polysomnography) allowing collection of neurophysiological sleep data in a manner not amenable to large-scale study. Sleep questionnaires will be used to assess general sleep quality and subjective sleep experience after each in-house recording. A motor sequencing task (MST) will be performed before and after the second night of polysomnography. In order to gather additional data about habitual sleep behaviour participants will be asked to wear a wrist worn activity monitor (actiwatch) and complete a sleep diary for two weeks.

Discussion: This study will explore the biological function of ZNF804A genotype (rs1344706) in healthy volunteers by examining detailed features of sleep architecture and physiology in relation to motor learning. Using a RBG approach will enable us to collect precise and detailed phenotypic data whilst achieving an informative biological gradient. It would not be feasible to collect such data in the large sample sizes that would be required under a random sampling scheme. By dissecting the role of individual variants associated with schizophrenia in this way, we can begin to unravel the complex genetic mechanisms of psychiatric disorders and pave the way for future development of novel therapeutic approaches.
\end{abstract}

Keywords: Sleep, Memory, Schizophrenia, Spindles, ZNF804A, rs1344706, Recall-by-genotype, ALSPAC

\footnotetext{
* Correspondence: Ullrich.Bartsch@bristol.ac.uk; laura.corbin@bristol.ac.uk

${ }^{\dagger}$ Equal contributors

'School of Physiology and Pharmacology, University of Bristol, Bristol, UK

${ }^{3}$ MRC Integrative Epidemiology Unit at University of Bristol, Bristol, UK

Full list of author information is available at the end of the article
} 


\section{Background}

Genome wide association studies (GWAS) and subsequent large-scale meta-analyses have been extremely successful in identifying large numbers of associations (>100) between common genetic variants and schizophrenia [1]. However, one limitation of this approach is that phenotypic precision is often sacrificed in favour of the larger sample sizes needed to override the power constraints that come from both the limitations to measurement and small genetic effects associated with common genetic variation. The reduction of complex traits such as schizophrenia to the binary classification of case/control in GWAS leaves much uncertainty around the exact role of many of the associated variants in the aetiology of disease.

In a GWAS of 479 patients with schizophrenia and 2937 controls (with replication of associated variants in a further 16,726 subjects) the strongest evidence for association was shown for rs1344706 (odds ratio [OR] $1.12, p=1.95 \times 10^{-7}$ ), a single nucleotide polymorphism (SNP) within the ZNF804A gene [2]. This finding has since been replicated in a number of other GWAS [1, 3-5] including a fine-mapping study that failed to detect any genetic variants within the ZNF804A locus that were more strongly associated with schizophrenia than rs1344706, which in this analysis had an OR for schizophrenia of 1.10 [1.07 - 1.14] [6].

Since its discovery, rs1344706 has been linked to a number of biological phenotypes [7]. The variant has been shown to correlate with altered neuroanatomy [8-11], abnormal neurophysiology [12, 13] and in particular changes in neurocognitive phenotypes [11, 14]. For example, researchers using functional magnetic imaging (fMRI) found evidence that healthy subjects carrying the rs1344706 risk variant showed changes in functional connectivity of the right dorsolateral prefrontal cortex during a working memory task $[15,16]$. Whilst cognitive defects are an established and wellstudied feature of schizophrenia [17, 18], more recently, links have been made between the cognitive symptom dimension and another feature of the condition - abnormal sleep patterns. Sleep disturbances have long been described in schizophrenia $[19,20]$ with studies consistently reporting altered sleep architecture where patients show increased sleep latency (time taken to fall asleep), decreased total sleep time and other altered sleep variables both on neuroleptic treatment and in the absence of medication [21, 22].

Sleep itself is a highly complex process that is tightly controlled by circadian rhythms and homeostatic mechanisms [23]. Sleep is known to be important for memory consolidation in both animals and humans [24, 25] with studies having shown improvements in both procedural and declarative memory consolidation following sleep [26]. Whilst even short naps lasting minutes to hours can result in better task performance compared to performance after an equally long period of wake time, longer periods of sleep lead to continued memory consolidation [27-29]. Moreover, sleep deprivation can severely impair memory consolidation [30]. The importance of sleep for memory consolidation in healthy participants is corroborated by impaired sleep-dependent memory consolidation in schizophrenia patients [31].

Human sleep can be classified into stages based on key features observed when monitoring brain activity (Electroencephalography; EEG), eye movement (Electrooculography; EOG) and muscle tone (Electromyography; EMG). The two main sleep stages are rapid eye movement (REM) sleep and non-rapid eye movement (NREM) sleep (further divided into stages 1-3) [32, 33]. In particular NREM sleep has been linked to memory consolidation in healthy volunteers [30]. More specifically, memory consolidation has been shown to correlate with spindle activity [34-36]. Spindle oscillations (a train of distinctive waves; 11$16 \mathrm{~Hz}, \geq 0.5 \mathrm{~s}$ ) are a feature found in the EEG during lighter NREM (stage 2) and arise from neuronal network oscillations spanning the cortex and thalamus. Spindle activity has also been shown to be reduced in patients with schizophrenia [37], and reduced spindle number and density correlated with impaired overnight improvement in a motor sequence task (MST) [38] (also in patients). Overall there is growing evidence that circuit abnormalities in schizophrenia link to changes in sleep physiology which in turn may contribute to the cognitive symptoms of schizophrenia [39, 40].

To the best of our knowledge the potential impact of variation in ZNF804A on sleep neurophysiology in patients with schizophrenia has not been characterised. The study of sleep phenotypes in patients with schizophrenia can be challenging, both in terms of recruitment of participants and study implementation. In many instances, issues around ethics, safety, and illness limit recruitment and bias samples to those with relatively mild disease severity. Further, results may be confounded by factors that are difficult to control, for example, medication. Where associations are observed, there are also issues around establishing the direction of causality. So far, efforts have been made to minimise limitations by recruiting medication-naïve participants [22] and by studying phenotypes in the first degree relatives of patients with schizophrenia [41]. We consider the value of an approach whereby the genetic control of phenotypes relevant to disease is investigated in healthy people, avoiding the aforementioned difficulties of working with patient groups.

Here we present a protocol designed to evaluate the efficacy of a recall-by-genotype (RBG) study design in 
investigating the impact of ZNF804A genotype (rs1344706) on sleep neurophysiology and memory consolidation in healthy subjects. In RBG studies, a sub-set of participants from an existing study are recruited on the basis of measured genotypic variation; existing biosamples can then be analysed or new data collected. This study has the potential to improve our understanding of both the genetic and neurophysiological basis of sleep and the biological basis for the association of ZNF804A variants with schizophrenia. In the longer term, linking sleep disturbances to cognitive deficits in psychiatric disorders could open up new avenues for the treatment of cognitive symptoms. Restoring normal sleep patterns in patients with psychiatric disorders may improve cognition, well-being and overall patient outcome [21].

\section{Methods/Design}

\section{Study design}

A RBG design will be implemented in a populationbased cohort. Participants will be recruited based on their genotype at rs1344706 and extended sleep-wake and cognitive testing datasets will be collected to allow detailed characterization of phenotypes.

\section{Ethical considerations and informed consent}

Ethical approval for the study has been obtained from the ALSPAC Ethics and Law Committee (ref. 9224). The data collection protocol has also previously been approved by The University of Bristol Faculty of Science Human Research Ethics Committee as part of a pilot study led by MWJ (ref. 8089). All participants will receive a participant information sheet prior to being recruited to the study and will be given the opportunity to ask questions both at the telephone screening and during the study nights. All participants will be asked to complete a written consent form on their first visit and they will be asked for continued consent verbally on their second visit. Participants will be free to withdraw from the study at any time. Participants will be appropriately reimbursed for their time and effort (as judged against other contemporary study initiatives) and all travel expenses will be reimbursed.

\section{Participant recruitment}

Male participants of European ancestry aged 21-23 years will be recruited from the Avon Longitudinal Study of Parents and Children (ALSPAC) on the basis of homozygous status at rs1344706. Homozygotes will be identified using existing genome-wide data, as imputed to the 1000 genomes reference panel (Phase 1, Version 3) (for more details on the genotyping and imputation of the cohort, see Additional file 1). ALSPAC is a transgenerational prospective birth cohort that began with the recruitment of 14,541 pregnant women resident in Avon, UK with expected dates of delivery 1st April 1991 to 31st December 1992. Since then, the health and development of mothers and their children has been followed across the life-course [42] (for more details about the cohort, see Additional file 1). The target sample size for this study will be 24, made up of 12 minor (CC) and 12 major (AA) homozygotes at rs1344706; in the event that the genotype groups are not equal, recruitment will continue until there is a minimum of 12 participants in each. Researchers will remain blind to participant genotype throughout the recruitment and data collection phases of the study.

Invitations will be sent to selected ALSPAC participants, together with a participant information sheet and reply slip, in batches of 100, with an even split between the two genotype groups until the target sample size is reached. Invitations will be sent preferentially to ALSPAC participants for whom other relevant phenotypic data is already available; these include WISC (Wechsler Intelligence Scale for Children) results, n-back task data and PLIKS (Psychosis-like symptoms) questionnaire data. An overview of the participant recruitment process is shown in Fig. 1. All participants who volunteer to take part will undergo a telephone screening to check eligibility against the following criteria:

\section{Inclusion criteria}

- In good physical or mental health;

- Non-smoker;

- Able to give informed consent as judged by the investigator;

- Available to attend two overnight clinic visits.

\section{Exclusion criteria}

- Previously diagnosed with a sleep disorder;

- Substantive current or past medical history;

- Taking medications that may affect or induce sleep;

- Working a regular night shift (or has done within the last six months);

- Current substance abuse, including alcohol excess (more than 24 units a week).

Participants will be asked to refrain from drinking alcohol in the $24 \mathrm{~h}$ prior to attending the clinic. They will also be advised not to consume any caffeinecontaining drinks after $4 \mathrm{pm}$ on the day of their visit to the clinic. Participants will be asked a series of screening questions at each visit to verify their adherence to these instructions and to confirm their on-going eligibility for the study. 


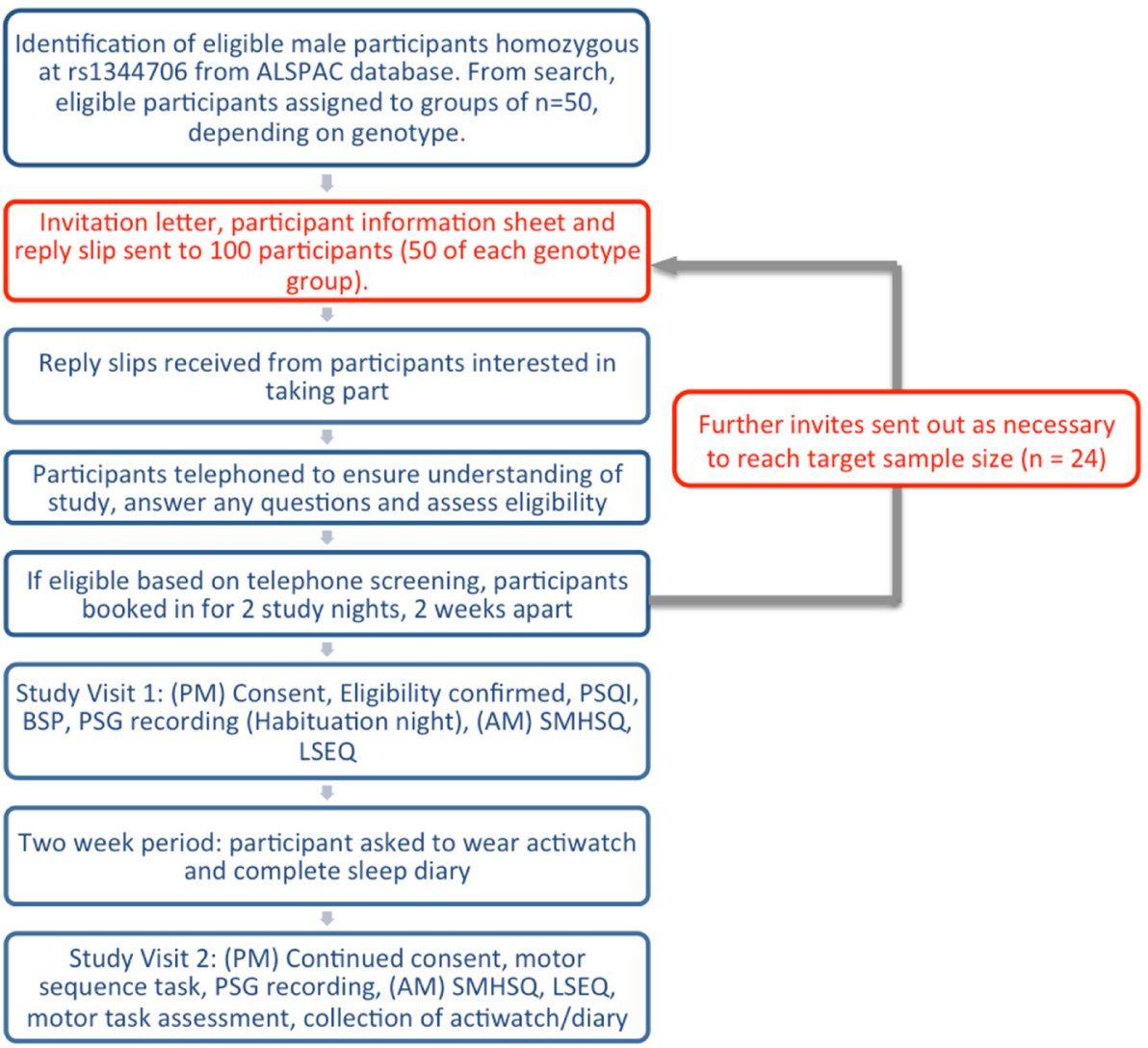

Fig. 1 Study Workflow. Tasks in red were carried out by ALSPAC staff (not researchers) in order that researchers remained blind to participant genotype (ALSPAC - Avon Longitudinal Study of Parents and Children; BSP - Bristol Sleep Profile; LSEQ - Leeds Sleep evaluation Questionnaire; PSG - polysomnography; PSQI -Pittsburgh Sleep Quality Index; SMH - St Mary's Hospital Sleep Questionnaire)

\section{Data collection}

The data collection phase for each participant will be two weeks long, beginning and ending with a night spent in the sleep suite at the clinic during which a polysomnography (PSG) will be performed.

\section{Actigraphy and sleep diary}

During their first visit to the clinic, the participants will be issued with a wrist worn activity monitor (MotionWatch 8, CamNtech, UK), which they will be asked to wear for the two weeks between overnight sleep monitoring nights. The MotionWatch contains a miniature accelerometer to allow measurement and recording of physical movement of the wrist, which provides a close correlation to whole body movement [43]. These data are stored into an internal memory and then downloaded for analysis at the end of the study period and analysed using MotionWare software [43]. Participants will also be asked to record information about their bedtime, wake/rise times, naps, daytime activities and caffeine and alcohol intake in a standardised diary for the duration of actigraphic monitoring. This will provide detailed information about the participant's habitual sleep behaviour with the diary information aiding interpretation of the actigraphy data. Both the MotionWatch and diary will be returned by the participant when they attend the clinic for the second time.

\section{Questionnaires}

Further behavioural data will be collected from each participant using a series of questionnaires. The Pittsburgh Sleep Quality Index (PSQI) [44] and Bristol Sleep Profile (BSP) [45] will be used at the first visit to assess the selfrated sleep quality and to indicate any specific sleep disturbance, respectively. Each participant will also be asked to complete the Edinburgh Handedness Inventory [46] which will be used to ascertain participant handedness ahead of the MST (see below). After each PSG recording, participants will be asked to complete the St Mary's Hospital Sleep Questionnaire (SMH) [47] and the Leeds Sleep Evaluation Questionnaire (LSEQ) [48]; to collect information about subjective experience of their night in the sleep laboratory, as compared to a normal night. 


\section{Polysomnography}

Each participant will stay in the sleep suite for two nights, one habituation night and one test night. A standard inlaboratory, overnight polysomnography (PSG), including video and audio recording, will be performed using Embla $^{\circ}$ sleep diagnostics equipment and RemLogic ${ }^{\mathrm{Tw}}$ software (Natus Medical Inc., California). Electrodes will be placed according to the internationally standardised 10 20 system and data acquired using a standard PSG recording montage. Additional electrodes will be placed to monitor eye movements, submental muscle activity and heart rate throughout the recording. Following electrode placement and bio-calibration, the participant will be able to follow their usual evening routine and will be encouraged to go to bed at their usual bedtime. The participant will be woken as close as possible to their usual wake time and the sensors removed. Following the completion of the data collection phase of the study, PSG data will be manually scored by an experienced expert, blinded to participant genotype, based on standardised criteria [32, 33]. A range of sleep variables will then be derived from the annotated PSG recordings (see Table 1).

\section{Motor sequence task}

On their second visit participants will perform a MST

[38] approximately two hours before they go to bed and again the following morning. Prior to completing the MST the participant will be asked to complete the Stanford Sleepiness Scale [49]. During the task participants will be shown a sequence of numbers consisting of 5 elements (4-1-2-3-4) on a computer. They will be asked to type out this sequence with their non-dominant hand as fast and as accurately as possible for $30 \mathrm{~s}$. Following this they will be given a $30 \mathrm{~s}$ break. This will be repeated 12 times.

\section{Data analysis}

A range of primary and secondary outcome measures will be derived from the raw data, as outlined in Table 1 . We plan to examine the difference in primary outcome measures across the recall strata, including a range of sleep variables and performance in the MST task.

\section{Discussion}

Here we describe a study to investigate the impact of sequence variation in ZNF804A on sleep neurophysiology and memory consolidation in healthy subjects. Existing research shows that the rs1344706 variant in ZNF804A is associated with schizophrenia and a range of neuroanatomical and neurocognitive phenotypes. It is also known that in patients with schizophrenia, abnormal sleep phenotypes are associated with poorer memory

Table 1 A summary of data collected and corresponding outcome measures

\begin{tabular}{|c|c|c|}
\hline & Data source & Outcome measures \\
\hline \multirow[t]{6}{*}{ Primary outcome measures } & \multirow[t]{3}{*}{ Polysomnography } & $\begin{array}{l}\text { Sleep variables derived from scored recordings, e.g. total sleep } \\
\text { time (TST), sleep onset latency (SOL), sleep efficiency (SE), } \\
\text { wake after sleep onset (WASO) }\end{array}$ \\
\hline & & $\begin{array}{l}\text { Time spent in each sleep stage; S1, S2, Slow wave sleep (SWS) } \\
\text { and rapid eye movement sleep (REM) }\end{array}$ \\
\hline & & $\begin{array}{l}\text { Spectral composition of EEG data (delta, theta, alpha, sigma and } \\
\text { beta frequency bands) }\end{array}$ \\
\hline & \multirow[t]{3}{*}{ Motor sequence task } & Number of correct sequences pre- and post-sleep \\
\hline & & Percentage improvement \\
\hline & & Reaction time pre- and post-sleep \\
\hline \multirow[t]{9}{*}{ Secondary outcome measures } & \multirow[t]{3}{*}{ Actigraphy } & $\begin{array}{l}\text { Sleep/Wake estimates e.g. sleep period, sleep efficiency, } \\
\text { total sleep time. }\end{array}$ \\
\hline & & $\begin{array}{l}\text { Non-parametric circadian rhythm analysis (NPCA) e.g. } 10 \text { most } \\
\text { active hours (M10), } 5 \text { least active hours (L5) }\end{array}$ \\
\hline & & Daily activity levels \\
\hline & Sleep diary & $\begin{array}{l}\text { Detailed information about rest/activity routine (to aid interpretation } \\
\text { of actigraphy) }\end{array}$ \\
\hline & Pittsburgh Sleep Quality Index & Sleep quality (over previous month) \\
\hline & Bristol Sleep Profile & Assessment for sleep disorders (eligibility) \\
\hline & St Mary's Hospital Sleep Questionnaire & Sleep quality, latency, continuity, satisfaction etc. (study nights) \\
\hline & Leeds Sleep Evaluation Questionnaire & $\begin{array}{l}\text { Total Score and individual components; Getting to sleep, quality } \\
\text { of sleep, awake following sleep, behaviours following awakening } \\
\text { (study nights) }\end{array}$ \\
\hline & Stanford Sleepiness Scale & Assessment of alertness prior to MST \\
\hline
\end{tabular}


consolidation during sleep. Building on this knowledge, this study aims to elucidate the aetiology of the association of rs1344706 with schizophrenia, in particular, whether the variant influences sleep neurophysiology and has a corresponding effect on memory consolidation.

The use of a RBG study design provides important advantages for this type of research question. It allows for more targeted selection of participants and as a result increases the efficiency of research and focuses the distribution of resources [50]. Participants, whose genotype has previously been determined, can be selected on the basis of having a certain genetic variant that is thought to contribute to a specific functional change. As a result fewer participants need to be recruited to achieve equivalent power to a similar population based study. In this study we will aim to recruit 24 participants, 12 in each homozygote genotype group. In comparison, based on a minor homozygote (CC) frequency for rs 1344706 of $17 \%$ in the ALSPAC cohort, approximately 70 individuals would need to be recruited and genotyped to ensure a similar number of participants in the minor homozygote group. This amounts to an approximate tripling of the study time required under the RBG model if a random sampling approach was used (from $\sim 480 \mathrm{~h}$ to $\sim 1400 \mathrm{~h}$ ).

This property of RBG is particularly important when the data collection procedure is particularly labour and/or time intensive as in this study. In this sense, a RBG design represents the antithesis of GWAS with sample size able to be sacrificed in favour of phenotypic precision, with the guaranteed biological gradient providing optimal statistical power. We also expect rs1344706 to show larger effects as a result of using indices of neurophysiological function rather than exclusively cognitive measures [14]. Furthermore, the RBG approach builds on the theoretical developments around causal inference derived from Mendelian randomization and as such avoids the issues of confounding and reverse causality that commonly effect traditional observational studies [51].

Whilst the use of a RBG study design provides a new and powerful approach to research into the effects of genetic variation on phenotypes, it also creates new ethical challenges that should be considered [52]. It is important to provide information to participants about why they have been invited to the study but at the same time not to disclose genetic information, especially when the clinical consequences of this information are unclear or unknown. When this study was conceived careful consideration was given to its ethical implications. Both the ALSPAC Ethics and Law Committee and the ALSPAC Original Cohort Advisory Panel (OCAP) were consulted on decisions regarding recruitment and the disclosure of information to participants. In addition impartial information will be collected on the ethical issues surrounding the study. Whilst no individual genetic information will be disclosed, participants will be informed that they were recruited on the basis of their genotype and they will be given opportunities to ask questions about the study and RBG recruitment method. Researchers will remain blind to participant genotype throughout the data collection phase of the study, with genotype information only being released following the anonymization of the study data.

In summary, a RBG study is described that will contribute to our understanding of the aetiology of variation in sleep neurophysiology and the impact of genetic variants on this. By exploiting the analytical gains afforded through extremely precise phenotyping over informative genetic strata, this approach can simultaneously be financially viable (due to relatively small sample sizes) and analytically efficient. This study will help to further understanding of the neurophysiology of sleep and sleep disturbances. Whilst the sleep phenotypes studied may not necessarily be linked directly to disease, they could nevertheless provide information on pre-morbid phenotypes and improve understanding of disease pathophysiology. Following the completion of this pilot study, the results will be used to inform the planning of further larger studies, which may also look at other genetic variants associated with schizophrenia.

\section{Additional file}

Additional file 1: The Avon Longitudinal Study of Parents and Children. This file contains a description of the cohort used for the study, including details of participant recruitment and genotyping and imputation procedures. (PDF $72 \mathrm{~kb}$ )

\section{Competing interests}

The authors declare that they have no competing interests. UB is employed by Eli Lilly, UK as part of a LIFA postdoctoral fellowship.

\section{Authors' contributions}

MWJ and NJT conceived and supervised the study. $\mathrm{CH}$ drafted the manuscript. CH, LC, UB, and CD will conduct the sleep studies. All authors read and approved the final manuscript.

\section{Acknowledgements}

$\mathrm{CH}$ is an academic foundation doctor supported by North Bristol NHS Trust. LJC and NJT are supported by the Medical Research Council MC UU 12013/3. MWJ is supported by the Medical Research Council (G1002064).

We gratefully acknowledge funding from the Elizabeth Blackwell Institute Catalyst Fund.

Avon Longitudinal Study of Parents and Children (ALSPAC): We are extremely grateful to all the families who took part in this study, the midwives for their help in recruiting them, and the whole ALSPAC team, which includes interviewers, computer and laboratory technicians, clerical workers, research scientists, volunteers, managers, receptionists and nurses. GWAS data for 9912 ALSPAC participants was generated by Sample Logistics and Genotyping Facilities at the Wellcome Trust Sanger Institute and LabCorp (Laboratory Corporation of America) using support from 23andMe. Please note that the study website contains details of all the data that is available through a fully searchable data dictionary (http://www.bris.ac.uk/ alspac/researchers/data-access/data-dictionary/). The UK Medical Research 
Council and the Wellcome Trust (Grant ref: 102215/2/13/2) and the University of Bristol provide core support for ALSPAC. This work was also supported by the Medical Research Council Integrative Epidemiology Unit (MC_UU_12013/3). This publication is the work of the authors and they will serve as guarantors for the contents of this paper.

\section{Author details}

${ }^{1}$ School of Physiology and Pharmacology, University of Bristol, Bristol, UK. ${ }^{2}$ Clinical Research and Imaging Centre (CRICBristol), University of Bristol, Bristol, UK. ${ }^{3}$ MRC Integrative Epidemiology Unit at University of Bristol, Bristol, UK.

\section{Received: 10 September 2015 Accepted: 20 October 2015} Published online: 24 October 2015

\section{References}

1. Ripke S, Neale BM, Corvin A, Walters JTR, Farh K-H, Holmans PA, et al. Biological insights from 108 schizophrenia-associated genetic loci. Nature. 2014;511(7510):421-7.

2. O'Donovan MC, Craddock N, Norton N, Williams H, Peirce T, Moskvina V, et al. Identification of loci associated with schizophrenia by genome-wide association and follow-up. Nat Genet. 2008;40(9):1053-5.

3. Riley B, Thiselton D, Maher BS, Bigdeli T, Wormley B, McMichael GO, et al. Replication of association between schizophrenia and ZNF804A in the Irish Case-control Study of Schizophrenia sample. Mol Psychiatry. 2010;15(1):29-37.

4. Purcell SM, Wray NR, Stone JL, Visscher PM, O'Donovan MC, Sullivan PF, et al. Common polygenic variation contributes to risk of schizophrenia and bipolar disorder. Nature. 2009;460(7256):748-52.

5. Steinberg $S$, Mors $O$, Borglum AD, Gustafsson O, Werge T, Mortensen PB, et al. Expanding the range of ZNF804A variants conferring risk of psychosis. Mol Psychiatry. 2011;16(1):59-66.

6. Williams HJ, Norton N, Dwyer S, Moskvina V, Nikolov I, Carroll L, et al. Fine mapping of ZNF804A and genome-wide significant evidence for its involvement in schizophrenia and bipolar disorder. Mol Psychiatry. 2011;16(4):429-41.

7. Sun Y, Hu D, Liang J, Bao Y-P, Meng S-Q, Lu L, et al. Association between variants of zinc finger genes and psychiatric disorders: Systematic review and meta-analysis. Schizophr Res. 2015;162(1-3):124-37.

8. Nenadic I, Maitra R, Basmanav FB, Schultz CC, Lorenz C, Schachtzabel C, et al. ZNF804A genetic variation ( $r$ 1344706) affects brain grey but not white matter in schizophrenia and healthy subjects. Psychol Med. 2015;45(1):143-52.

9. Schultz CC, Nenadic I, Riley B, Vladimirov VI, Wagner G, Koch K, et al. ZNF804A and Cortical Structure in Schizophrenia: In Vivo and Postmortem Studies. Schizophr Bull. 2014;40(3):532-41.

10. Wassink TH, Epping EA, Rudd D, Axelsen M, Ziebell S, Fleming FW, et al. Influence of ZNF804a on Brain Structure Volumes and Symptom Severity in Individuals With Schizophrenia. Arch Gen Psychiatry. 2012;69(9):885-92.

11. Gurung R, Prata DP. What is the impact of genome-wide supported risk variants for schizophrenia and bipolar disorder on brain structure and function? A systematic review. Psychol Med. 2015;1-20.

12. Paulus FM, Krach S, Bedenbender J, Pyka M, Sommer J, Krug A, et al. Partial support for ZNF804A genotype-dependent alterations in prefrontal connectivity. Hum Brain Mapp. 2013;34(2):304-13.

13. del Re EC, Bergen SE, Mesholam-Gately RI, Niznikiewicz MA, Goldstein JM, Woo TU, et al. Analysis of schizophrenia-related genes and electrophysiological measures reveals ZNF804A association with amplitude of P300b elicited by novel sounds. Translational Psychiatry. 2014;4(1):e346.

14. Rose EJ, Donohoe G. Brain vs Behavior: An Effect Size Comparison of Neuroimaging and Cognitive Studies of Genetic Risk for Schizophrenia. Schizophr Bull. 2013;39(3):518-26.

15. Esslinger C, Walter H, Kirsch P, Erk S, Schnell K, Arnold C, et al. Neural Mechanisms of a Genome-Wide Supported Psychosis Variant. Science. 2009:324(5927):605.

16. Esslinger C, Kirsch P, Haddad L, Mier D, Sauer C, Erk S, et al. Cognitive state and connectivity effects of the genome-wide significant psychosis variant in ZNF804A. Neuroimage. 2011;54(3):2514-23.

17. Mohamed S, Paulsen JS, O'Leary D, Arndt S, Andreasen N. Generalized cognitive deficits in schizophrenia - A study of first-episode patients. Arch Gen Psychiatry. 1999;56(8):749-54.
18. Green MF, Kern RS, Heaton RK. Longitudinal studies of cognition and functional outcome in schizophrenia: implications for MATRICS. Schizophr Res. 2004;72(1):41-51

19. Monti JM, Monti D. Sleep in schizophrenia patients and the effects of antipsychotic drugs. Sleep Med Rev. 2004;8(2):133-48.

20. Cohrs S. Sleep disturbances in patients with schizophrenia: impact and effect of antipsychotics. CNS Drugs. 2008;22(11):939-62.

21. Tandon R, Shipley JE, Taylor S, Greden JF, Eiser A, DeQuardo J, et al. Electroencephalographic Sleep Abnormalities in Schizophrenia Relationship to Positive/Negative Symptoms and Prior Neuroleptic Treatment. Arch Gen Psychiatry. 1992;49(3):185-94.

22. Chouinard S, Poulin J, Stip E, Godbout R. Sleep in untreated patients with schizophrenia: A meta-analysis. Schizophr Bull. 2004;30(4):957-67.

23. Borbely AA, Achermann P. Sleep homeostasis and models of sleep regulation. J Biol Rhythms. 1999;14(6):557-68.

24. Stickgold R. Sleep-dependent memory consolidation. Nature. 2005;437(7063):1272-8.

25. Eschenko O, Moelle M, Born J, Sara SJ. Elevated sleep spindle density after learning or after retrieval in rats. J Neurosci. 2006;26(50):12914-20.

26. Rasch B, Born J. About Sleep's Role in Memory. Physiol Rev. 2013;93(2):681-766

27. Walker MP, Brakefield T, Seidman J, Morgan A, Hobson JA, Stickgold R. Sleep and the time course of motor skill learning. Learn Mem. 2003;10(4):275-84

28. Stickgold R, James $L$, Hobson JA. Visual discrimination learning requires sleep after training. Nat Neurosci. 2000;3(12):1237-8.

29. Diekelmann S, Born J. The memory function of sleep. Nat Rev Neurosci. 2010;11(2):114-26.

30. Walker MP, Brakefield T, Morgan A, Hobson JA, Stickgold R. Practice with sleep makes perfect: Sleep-dependent motor skill learning. Neuron. 2002;35(1):205-11.

31. Manoach DS, Stickgold R. Does abnormal sleep impair memory consolidation in schizophrenia? Front Hum Neurosci. 2009;3:21.

32. Iber C, Ancoli-Israel S, Chesson A, Quan S. The AASM Manual for the Scoring of Sleep and Associated Events: Rules, Terminology and Technical Specifications. 1st ed. Westchester: American Academy of Sleep Medicine; 2007.

33. Silber MH, Ancoli-Israel S, Bonnet MH, Chokroverty S, Grigg-Damberger MM, Hirshkowitz M, et al. The visual scoring of sleep in adults. J Clin Sleep Med. 2007;3(2):121-31.

34. Marshall L, Helgadottir $H$, Moelle M, Born J. Boosting slow oscillations during sleep potentiates memory. Nature. 2006;444(7119):610-3.

35. Moelle M, Eschenko O, Gais S, Sara SJ, Born J. The influence of learning on sleep slow oscillations and associated spindles and ripples in humans and rats. Eur J Neurosci. 2009;29(5):1071-81.

36. Gais S, Molle M, Helms K, Born J. Learning-dependent increases in sleep spindle density. J Neurosci. 2002;22(15):6830-4

37. Ferrarelli F, Huber R, Peterson MJ, Massimini M, Murphy M, Riedner BA, et al. Reduced sleep spindle activity in schizophrenia patients. Am J Psychiatr. 2007;164(3):483-92.

38. Wamsley EJ, Tucker MA, Shinn AK, Ono KE, McKinley SK, Ely AV, et al. Reduced Sleep Spindles and Spindle Coherence in Schizophrenia: Mechanisms of Impaired Memory Consolidation? Biol Psychiatry. 2012;71(2):154-61.

39. Gardner RJ, Kersante F, Jones MW, Bartsch U. Neural oscillations during non-rapid eye movement sleep as biomarkers of circuit dysfunction in schizophrenia. Eur J Neurosci. 2014;39(7):1091-106.

40. Wulff K, Gatti S, Wettstein JG, Foster RG. Sleep and circadian rhythm disruption in psychiatric and neurodegenerative disease perspectives. Nat Rev Neurosci. 2010;11(8):589-99.

41. Manoach DS, Demanuele C, Wamsley EJ, Vangel M, Montrose DM, Miewald J, et al. Sleep spindle deficits in antipsychotic-naive early course schizophrenia and in non-psychotic first-degree relatives. Front Hum Neurosci. 2014;8:762.

42. Boyd A, Golding J, Macleod J, Lawlor DA, Fraser A, Henderson J, et al. Cohort Profile: The "Children of the 90s" - the index offspring of the Avon Longitudinal Study of Parents and Children. Int J Epidemiol. 2013;42(1):11127.

43. CamNtech. The MotionWatch 8 and MotionWare User Guide. http:// www.camntech.com/products/motionwatch/motionwatch-8-overview. Accessed 01/09/2015. 
44. Buysse DJ, Reynolds CF, Monk TH, Berman SR, Kupfer DJ. The Pittsburgh Sleep Quality Index - A New Instrument For Psychiatric Practice And Research. Psychiatry Res. 1989;28(2):193-213.

45. Smith A, Rich N, Wilson S, Nutt D. Subjective and Objective Assessment of the Effects of Noise, Noise Sensitivity and Noise Disturbed Sleep on Health Presented at: InterNoise01 - the 2001 International Congress on Noise Control Engineering, The Hague, The Netherlands, 28-30 August 2001. INTER-NOISE and NOISE-CON Congress and Conference Proceedings, InterNoise01, The Hague, The Netherlands. Indianapolis, IN: INCE - Institute of Noise Control Engineering; 1171-76.

46. Oldfield RC. The Assessment And Analysis Of Handedness: The Edinburgh Inventory. Neuropsychologia. 1971;9(1):97-113.

47. Leigh TJ, Bird HA, Hindmarch I, Constable PDL, Wright V. Factor-Analysis Of The St-Marys-Hospital Sleep Questionnaire. Sleep. 1988;11(5):448-53.

48. Hindmarch I. 1,4-Benzodiazepine, Temazepam (K 3917), Its Effect On Some Psychological Parameters Of Sleep And Behavior. Arzneimittel-Forschung/ Drug Res. 1975;25(11):1836-9.

49. Hoddes E, Zarcone V, Smythe H, Phillips R, Dement WC. Quantification of sleepiness - New approach. Psychophysiology. 1973;10(4):431-6.

50. Ware JJ, Timpson N, Smith GD, Munafo MR. A recall-by-genotype study of CHRNA5-A3-B4 genotype, cotinine and smoking topography: study protocol. BMC Med Genet. 2014;15(1):13.

51. Davey Smith G, Ebrahim S. What can mendelian randomisation tell us about modifiable behavioural and environmental exposures? Br Med J. 2005;330(7499):1076-9.

52. Beskow LM, Fullerton SM, Namey EE, Nelson DK, Davis AM, Wilfond BS Recommendations for ethical approaches to genotype-driven research recruitment. Hum Genet. 2012;131(9):1423-31.

\section{Submit your next manuscript to BioMed Central and take full advantage of:}

- Convenient online submission

- Thorough peer review

- No space constraints or color figure charges

- Immediate publication on acceptance

- Inclusion in PubMed, CAS, Scopus and Google Scholar

- Research which is freely available for redistribution 